\title{
What is the Importance of Clinical Clues, and How can we Avoid Mistakes in Following Them?
}

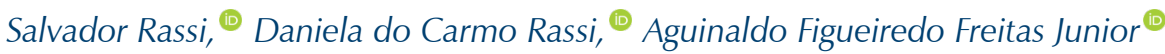 \\ Faculdade de Medicina da UFG, Goiânia, GO - Brazil
}

Approximately half of patients with heart failure (HF) have preserved ejection fraction (EF), ${ }^{1}$ and approximately half of these patients have increased left ventricular (LV) wall thickness ${ }^{2}$. This increase in LV wall thickness is usually attributed to left ventricular hypertrophy induced by arterial hypertension $(\mathrm{AH})$. However, cardiac amyloid fibril infiltration also increases ventricular wall thickness, and it can lead to a clinical syndrome of HF with preserved EF (HFpEF). ${ }^{3}$

Light chain amyloidosis (AL) cardiomyopathy occurs in patients with $A L$ or primary amyloidosis, which is a hematologic disease with specific hematologic treatment. Both hereditary transthyretin (hATTR) and wild-type (ATTRwt) amyloidosis can cause transthyretin amyloid cardiomyopathy (ATTR-CM). ${ }^{3}$

The identification of ATTR-CM, among the broad spectrum of the population with HFpEF, is particularly important, because there is currently a specific, effective treatment for these clinical forms of HFpEF. ${ }^{4}$ These findings and clinical observations are extremely important, and they alert us that we are possibly facing a patient with cardiac amyloidosis.

What is the prevalence of ATTR-CM in the population with HFpEF with a thickened ventricular wall?

In a recently published prospective cohort study ${ }^{5}$ involving 1235 consecutive patients with validated diagnosis of HF and increased risk of ATTR-CM due to age, HFpEF, and increased LV thickness, the prevalence by clinical recognition was only $1.3 \%$, but it was approximately six times higher $(6.3 \%)$ in the sample of 286 patients who had signed informed consent for additional tests for systematic screening of ATTR-CM. This evaluation consisted of technetium 99m pyrophosphate scintigraphy and appropriate laboratory exams for ruling out AL amyloidosis.

Several clinical parameters help us consider amyloidosis; Table 1 systematizes the usual clinical clues. ${ }^{6}$

The prevalence is usually higher in men $(80 \%)$, and it increases with age. History of carpal tunnel syndrome (37\%) and spinal stenosis (31\%) are common. ${ }^{5}$ These extra-cardiac alterations may precede the development of cardiac amyloidosis

\section{Keywords}

Heart Failure; Amyloidosis; Restrictive Cardiomyopathy.

Mailing Address: Salvador Rassi •

Rua T-36, 3229. Postal Code 74223-044, Setor Bueno, Goiânia, GO - Brazil. E-mail: srassi@cardiol.br

Manuscript received September 09, 2021, revised manuscript September 24, 2021, accepted September 24, 2021.

DOI: https://doi.org/10.36660/abchf.20210020 by many years. The recognition of these signs as part of the clinical picture of amyloidosis is essential, and it may lead to earlier diagnosis and prevent the progression of heart disease through the implementation of specific treatment.

In cases where peripheral neuropathy affects the most distal segments of the limbs, especially the lower limbs, and progresses to involve the proximal and upper limbs, this is a clinical clue that should lead us to consider amyloidosis. ${ }^{6}$

Eelectrocardiogram is usually the first exam that is requested for evaluation of a possible heart disease. In AL cardiac amyloidosis, low voltage of the QRS complex calls our attention, especially if we have already conducted an echocardiogram showing significant left ventricular hypertrophy. In the ATTR form, this finding is less common, occurring in approximately $30 \%$ of cases. Therefore, this discrepancy between the magnitude of hypertrophy on the echocardiogram and the QRS amplitude on the electrocardiogram is a clinical clue that should be emphasized in considering diagnosis of cardiac amyloidosis. The rhythm of atrial fibrillation is frequent, due to the large atrial volumes caused by the elevated LV end-diastolic pressure (restrictive cardiomyopathy). ${ }^{7}$

Echocardiography is one of the most important tests to raise suspicion of cardiac amyloidosis. We should consider the hypothesis of amyloidosis when we find LV wall thickening (greater than $12 \mathrm{~mm}$ ) in the absence of $\mathrm{AH}$, in elderly patients ( $>65$ years), and in patients with biatrial enlargement that is disproportionate to the size of the ventricles. Also noteworthy is the thickening of the atrioventricular valves and the interatrial septum, as well as increased echogenicity of the myocardium with a granular appearance, which are alterations that are due to the deposition of amyloid filaments in these structures ${ }^{7}$ (Table 2 and Figure 1).

It is in these "combined clues" that our greatest likelihood of diagnosing cardiac amyloidosis lies. When we are faced with an elderly patient, with clinical picture of HF, non-dilated LV, preserved EF, and thickened LV walls, without $\mathrm{AH}$, this hypothesis is imposed. Another interesting clue that needs to be taken into account is when the patient presents with a diagnosis of late-onset hypertrophic cardiomyopathy, after 60 years of age. This is unusual, and it should lead us to remember cardiac amyloidosis. ${ }^{7}$

Another clinical situation is that of a patient with aortic stenosis, with thickening of the right ventricular walls, particularly in paradoxical cases with low flow and low gradient.

Therefore, when faced with diagnosis of HFpEF, with symptoms of dysfunction in another organ (peripheral sensorimotor neuropathy, gastrointestinal and central nervous system manifestations, carpal tunnel syndrome, 


\section{Viewpoint}

\section{Table 1 - Clues related to history and physical examination}

HFpEF, especially in elderly men (> 65 years)

Intolerance to ACEI/ARB/ARNI/BB

Bilateral carpal tunnel syndrome

Biceps tendon rupture

Peripheral polyneuropathy and/or autonomic dysfunction

Periorbital ecchymosis

Macroglossia

ACEl: angiotensin converting enzyme inhibitors; ARB: angiotensin receptor blocker; ARNI: angiotensin receptor-neprilysin inhibitor; BB: betablockers;

HFpEF: heart failure with preserved ejection fraction.

\section{Table 2 - Clues related to routine imaging exams}

Concentric thickening of the LV walls with reduced QRS amplitude

Echocardiogram showing hypertrophy of the septum and LVPW, biventricular hypertrophy, valve thickening, pericardial effusion, or interatrial septal thickening; myocardial hyperrefringence

Reduced longitudinal strain that spares the apical region (apical sparing)

Restrictive pattern of LV filling with thickening of the RV walls

Delayed enhancement on CMR with diffuse subendocardial or transmural pattern or increased extracellular volume

CMR: cardiac magnetic resonance; LV: left ventricle; LVPW: left ventricular posterior wall; QRS: QRS complex on electrocardiogram; RV: right ventricle.

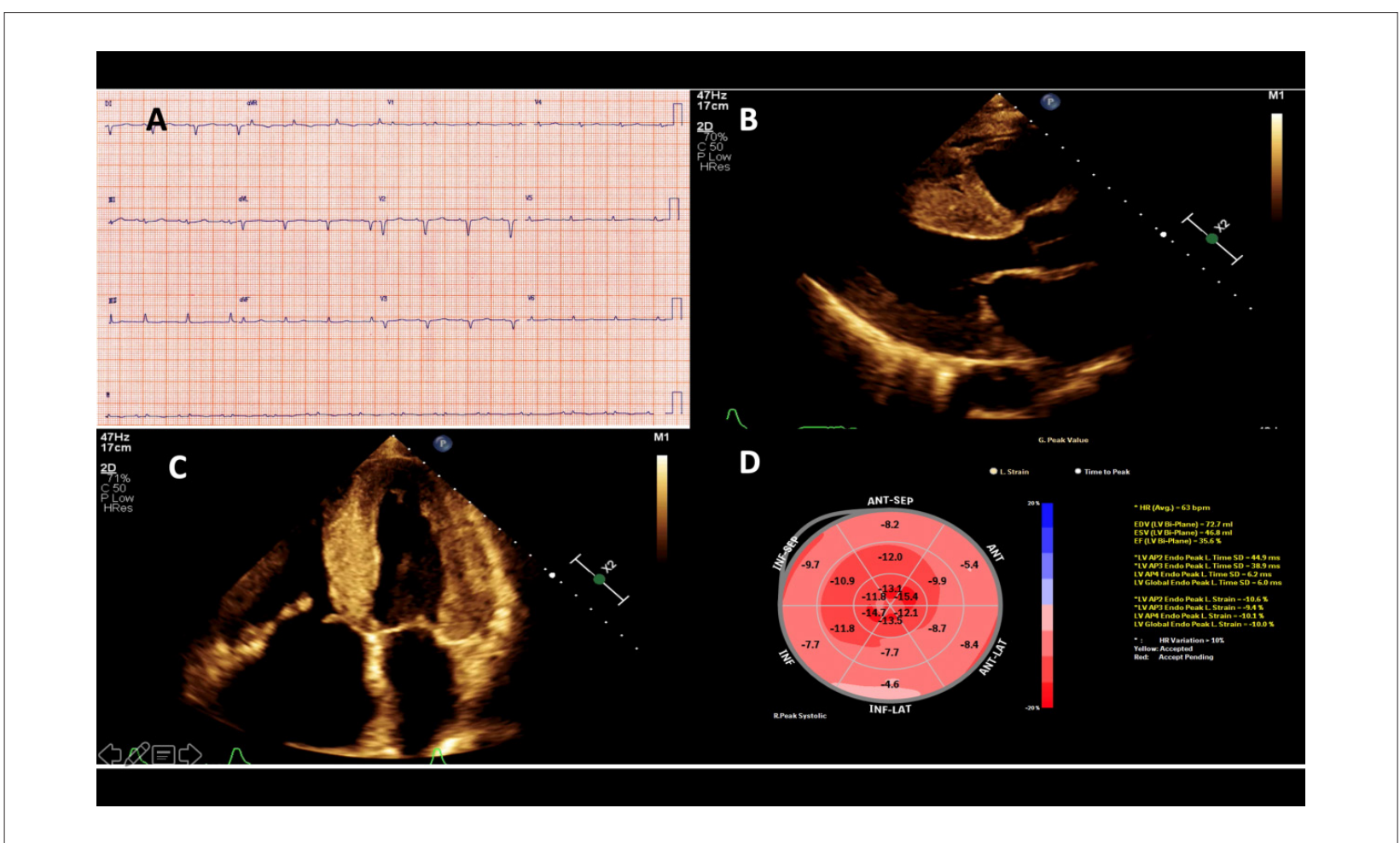

Figure 1 - Illustrative image shows electrocardiogram (A) with low QRS voltage. Transthoracic echocardiogram demonstrates, through the parasternal long axis $(B)$ and apical 4-chamber (C) views, increased myocardial thickness with a shiny, granular appearance, thickening of the atrioventricular valve cusps, and significant dilation of both atria. In the parametric representation of longitudinal systolic strain (D), it is possible to observe reduced global longitudinal strain with a characteristic apical sparing pattern (images from the authors' personal archives). 
Viewpoint

Table 3 - Combined clues

HF with non-dilated LV and septum $>12 \mathrm{~mm}$, in patients without $\mathrm{AH}$

Clinical presentation of late-onset HCM (> 60 years)

AoS with thickening of the RV walls, especially in paradoxical cases with low flow and low gradient

AH: arterial hypertension; AoS: aortic stenosis; HCM: hypertrophic cardiomyopathy; HF: heart failure; LV: left ventricle; RV: right ventricle.

and autonomic neuropathy, mainly) in a patient over 65 years of age, who is also unresponsive to the usual treatment for $\mathrm{HF}$, we must consider the possibility of cardiac amyloidosis. ${ }^{8}$

The implementation of this systematic evaluation can increase the diagnosis of ATTR-CM and make appropriate treatment possible for this HFpEF phenotype.

\section{Author contributions}

Conception and design of the research: Rassi S, Rassi DC, Freitas AF. Acquisition of data: Rassi S, Rassi DC, Freitas AF. Analysis and interpretation of the data: Rassi S, Rassi DC, Freitas AF. Statistical analysis:Rassi S. Writing of the manuscript:Rassi S, Rassi DC, Freitas AF. Critical revision of the manuscript for intellectual content:Rassi S.

\section{References}

1. Redfield MM. Heart Failure with Preserved Ejection Fraction. N Engl J Med. 2017;376(9):897. doi: 10.1056/NEJMc1615918.

2. Mohammed SF, Borlaug BA, Roger VL, Mirzoyev SA, Rodeheffer RJ, Chirinos JA, etal. Comorbidity and Ventricular and Vascular Structure and Function in Heart Failure with Preserved Ejection Fraction: A Community-Based Study. Circ Heart Fail. 2012;5(6):710-9. doi: 10.1161/CIRCHEARTFAILURE.112.968594.

3. Ruberg FL, Grogan M, Hanna M, Kelly JW, Maurer MS. Transthyretin Amyloid Cardiomyopathy: JACC State-of-the-Art Review. J Am Coll Cardiol. 2019;73(22):2872-91. doi: 10.1016/j.jacc.2019.04.003.

4. MaurerMS, SchwartzJH, Gundapaneni B, Elliott PM, Merlini G, WaddingtonCruz M, et al. Tafamidis Treatment for Patients with Transthyretin Amyloid Cardiomyopathy. N Engl J Med. 2018;379(11):1007-16. doi: 10.1056/ NEJMoa1805689.

\section{Potential Conflict of Interest}

No potential conflict of interest relevant to this article was reported.

\section{Sources of Funding}

There were no external funding sources for this study.

\section{Study Association}

This study is not associated with any thesis or dissertation work.

\section{Ethics approval and consent to participate}

This article does not contain any studies with human participants or animals performed by any of the authors.

5. AbouEzzeddine OF, Davies DR, Scott CG, Fayyaz AU, Askew JW, McKie PM, et al. Prevalence of Transthyretin Amyloid Cardiomyopathy in Heart Failure with Preserved Ejection Fraction. JAMA Cardiol. 2021:e213070. doi: 10.1001/jamacardio.2021.3070.

6. Simões MV, Fernandes F, Marcondes-Braga FG, Scheinberg P, Correia EB, Rohde LEP, et al. Posicionamento sobre Diagnóstico e Tratamento da Amiloidose Cardíaca - 2021. Arq. Bras. Cardiol. 2021;117(3):561-98. doi: 10.36660/abc.20210718.

7. Simões MV, Alves SMM, Fernandes F, Coelho Filho OR, Mangini S. Emerging Topics in Heart Failure: New Paradigms in Cardiac Amyloidosis. Arq Bras Cardiol. 2020;115(5):945-8. doi: 10.36660/abc.20201126.

8. Gertz MA. Hereditary ATTR Amyloidosis: Burden of Illness and Diagnostic Challenges. Am J Manag Care. 2017;23(7):107-12. 